\title{
Discovery of a high velocity, spatially extended emission "shell" in front of the southeast lobe of the $\eta$ Carinae Homunculus ${ }^{\star}$
}

\author{
D. G. Currie $^{1,2}$, B. N. Dorland ${ }^{1,3}$, and A. Kaufer ${ }^{4}$ \\ 1 Physics Department, University of Maryland, College Park, MD 20742-4111, USA \\ 2 European Southern Observatory, Garching bei Muenchen, Germany \\ 3 Astrometry Department, United States Naval Observatory, 3450 Mass. Ave, NW, Washington, \\ DC 20392-5420, USA \\ 4 European Southern Observatory, Alonso de Cordova 3107, Vitacura, Santiago 19, Chile
}

Received 2 April 2002 / Accepted 31 May 2002

\begin{abstract}
We report the discovery of the $\eta$ Carinae "Ghost Shell," a high-velocity, spatially extended emission feature that lies in front of the southeast lobe of the $\eta$ Carinae Homunculus. Using data obtained with "Kueyen," one of the European Southern Observatory's Very Large Telescope $8.2 \mathrm{~m}$ telescopes and its Ultraviolet and Visible Echelle Spectrograph instrument, we have observed a structure in velocity space of width $\approx 35 \mathrm{~km} \mathrm{~s}^{-1}$ and with Doppler velocities ranging from $-675 \leq v \leq-850 \mathrm{~km} \mathrm{~s}^{-1}$. This is up to $500 \mathrm{~km} \mathrm{~s}^{-1}$ faster than the Homunculus front wall. The structure is distinct from the front wall in velocity space, and extends beyond the Homunculus' spatial boundaries. The Ghost Shell has been detected in emission for multiple allowed Balmer lines and in forbidden lines of [NII], [SII], and [ArIII]. The feature is also associated with a complex absorption structure in $\mathrm{CaH}$ and $\mathrm{K}$ lines. We propose that the Ghost Shell lies outside the Homunculus and represents the forward shock between the fast stellar wind of the Great Eruption epoch and the older slow massive stellar wind.
\end{abstract}

Key words. stars: individual: $\eta$ Carinae - variables: LBV - winds, outflows - circumstellar matter

\section{Introduction}

The star $\eta$ Carinae, generally considered an extreme member of the Luminous Blue Variables (LBVs) (Humphreys \& Davidson 1994), and its surrounding nebulosities have been an ongoing subject of observation and theoretical modeling for many years. Much attention has been paid to the Homunculus nebula, whose material was expelled from the central star during the Great Eruption of c.1842 (Currie et al. 1996). Under the assumption of unaccelerated expansion of the Homunculus material, the planeof-sky and Doppler motion measurements have allowed us to define in detail the "Double Flask" Model of the Homunculus morphology (Dowling 1996; Currie et al. 2000). In order to refine the model, we are currently engaged in extending the above Doppler measurements using the high spectral resolution of the Ultraviolet and Visible Echelle Spectrograph (UVES), the excellent seeing conditons available at Paranal and the collection capability of the Very Large Telescope (VLT). These results represent

Send offprint requests to: B. N. Dorland,

e-mail: bdorland@usno.navy.mil

* Based on observations collected at the European Southern Observatory at Paranal, Chile (UVES commissioning II). the first use of UVES for extended objects such as the Homunculus.

During this effort to take advantage of the capabilities available with UVES, we have found a new emission feature. This feature, which we call the "Ghost Shell" (GS), extends at least over the southeast lobe of the Homunculus, with typical velocities of order hundreds of $\mathrm{km} \mathrm{s}^{-1}$ faster than the Homunculus front wall. Observational results described below lead us to conclude that the GS is a phenomenon associated with the Great Eruption, but distinct from the Homunculus. In this letter we describe the initial results relating to the GS and propose a physical explanation.

\section{Observations and reduction}

\subsection{Collection, calibration, and initial reduction}

UVES observations of $\eta$ Carinae were made in December 1999 and January 2000 using Kueyen, one of the $8.2 \mathrm{~m}$ VLT telescopes. The January 2000 data set consisted of 18 long slit spectra observations. Each slit's long axis was $10^{\prime \prime}$ (in the "blue" arm) or 12 " (in the "red" arm) wide. Each long slit was oriented orthogonal to (i.e, at a position angle $=45 \mathrm{deg}$ ) and centered on the Homunculus 


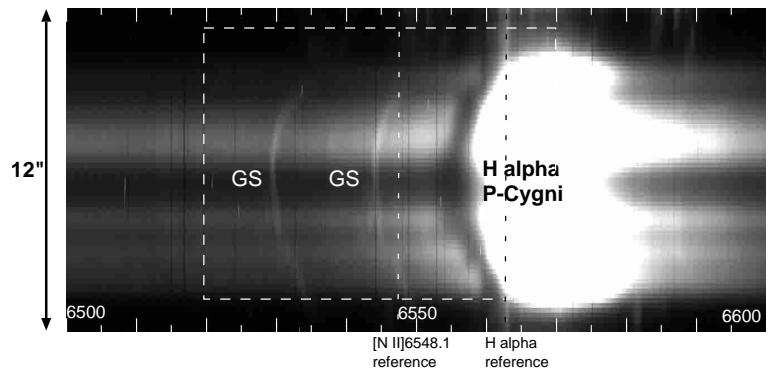

Fig. 1. $[\mathrm{NII}]$ and $\mathrm{H} \alpha$ emission in UVES data, slit -8. "GS" indicates suspected Ghost Shell emission. Dashed lines indicate reference wavelengths; box indicates region shown in Fig. 2. Abscissa is the spectral axis, ordinate is spatial.

axis of symmetry (AOS). We restrict discussion in this letter to slits covering the SE lobe of the Homunculus, numbered -2 to -8 , with the slit number corresponding (approximately) to offset in arc seconds from the central star position. The spectral coverage in the 2000 data taken in dichroic \#1 mode was 3100-3866 $\AA$ (blue) and $4791-6798 \AA$ (red); resolving power $\lambda / \delta \lambda \approx 70000$ (blue) and $\lambda / \delta \lambda \approx 100000$ (red); seeing for the observing session was $\approx 0.6^{\prime \prime}$ (much greater than the slit widths of $0.4^{\prime \prime}$ (blue) and $0.3^{\prime \prime}$ (red)); and exposure time in each slit position was 300 seconds. 1999 data were restricted to two slits, one in dichroic \#1 mode and the second in dichroic \#2 mode with resultant additional spectral coverage from 3760-4973 A (blue) and 6716-10 $401 \AA$ (red). 1999 exposure times were $3-5$ times longer than 2000 exposure times. All references in this paper are to 2000 data unless otherwise noted. Calibration and initial reduction was performed at the ESO Vitacura facility with the ESOMIDAS data reduction system using modified versions of the uves and feros contexts. The latter was modified in order to properly address the spatially extended nature of the source. The resultant combined order data has a spectral scale of $0.02 \AA$ and a spatial scale of $0.182^{\prime \prime}$ (blue) and $0.246^{\prime \prime}$ (red) per pixel.

\subsection{Analysis reduction}

Data reduction was accomplished as follows. A 2 D $3 \times 3$ median filter was applied to the data to reduce high frequency pixel noise. These data were then filtered with a $4 \AA$ wide median filter in the spectral direction. The latter was subtracted from the above data to remove low frequency spectral features and isolate GS lines. A Gaussian function was fitted to suspected emission line positions and centroid, width, and amplitude of these lines were calculated. From these results, we derived velocity, width in physical units, and "energy", defined as the product of the width of a line in $\mathrm{km} \mathrm{s}^{-1}$ by the amplitude as measured in relative flux units. In certain (noted) instances we summed over the spectral dimension to increase signal-tonoise ratio $(S / N)$.
Table 1. Ghost Shell emission line observations and strengths. " $\lambda_{0}$ " indicates the zero-velocity laboratory reference wavelength. "2000 Data" and "1999 Data" columns indicate slit offsets in which the line is detectable. $\left\langle E_{\text {norm }}\right\rangle$ column gives the mean line energy, normalized to $\mathrm{H} \beta=1.00$.

\begin{tabular}{lcccc}
\hline \hline Identification & $\lambda_{0}$ & 2000 Data & 1999 Data & $\left\langle E_{\text {norm }}\right\rangle$ \\
\hline $\mathrm{H} \gamma$ & 4340.5 & & -3 & 0.62 \\
$\mathrm{H} \beta$ & 4861.3 & $-8 \leftrightarrow-4$ & $-4,-3$ & 1.00 \\
{$[\mathrm{NII}]$} & 6548.1 & $-8 \leftrightarrow-2$ & -4 & 1.85 \\
$\mathrm{H} \alpha$ & 6562.8 & $-8 \leftrightarrow-5$ & -4 & 2.57 \\
{$[\mathrm{NII}]$} & 6583.5 & -7 & & 4.23 \\
{$[\mathrm{SII}]$} & 6716.4 & $-8 \leftrightarrow-6$ & -4 & 0.16 \\
{$[\mathrm{SII}]$} & 6730.8 & $-8 \leftrightarrow-6$ & -4 & 0.19 \\
{$[\mathrm{ArIII}]$} & 7135.7 & & -3 & 0.12 \\
\hline
\end{tabular}

\section{Observational results}

The initial discovery of the GS was made when measuring two correlated features observed in Slit -8 at about 6530 and $6544 \AA$, shown in Fig. 1. Referencing these two features to zero-velocity wavelengths of 6548.1 and $6562.8 \AA$ (i.e, $[\mathrm{NII}]$ and $\mathrm{H} \alpha$ ) yields a Doppler velocity of $\approx-825 \mathrm{~km} \mathrm{~s}^{-1}$ for both of the features.

According to the current Homunculus models and observations (Dowling 1996; Currie et al. 2000), blue-shifted photons must all be from wall emission, but no known Homunculus feature is present at this position in velocity space. For slit -8 , the front wall of the Homunculus is closer to $350 \mathrm{~km} \mathrm{~s}^{-1}$ (Currie et al. 2000; Davidson et al. 2001). In order to determine if this was an anomalous observation or an actual physical phenomenon, we searched for this feature in other slits and spectral regions. Using the velocity space profile for [NII] 6548.1 as a template, we have detected the GS feature in emission in eight lines in multiple slit positions in both the 1999 and 2000 data (see Table 1).

Representative data for five of the lines present in the 2000 data are shown in Fig. 2. The data have been scaled and aligned so that the abscissa represents velocity with respect to three of the reference wavelengths given in Table 1. The unmistakable agreement in Doppler velocities of the lines is excellent. Velocity measurements on a spatial row-by-spatial row basis typically differ by $<1 \%$. An example of this close agreement in line velocities for five different lines are shown in Fig. 3 for slit -7 .

\section{Discussion}

\subsection{3-D Spatial configuration of Ghost Shell}

The definitive method to determine the 3-D spatial configuration of the GS is to combine plane-of-the-sky astrometry with Doppler astrometry (i.e., the method used for the Homunculus described in Dowling 1996 and Currie et al. 2000). However, we have essentially a single epoch so other methods must be used to evaluate the structure of the GS. We hypothesize that the form of the GS is roughly 


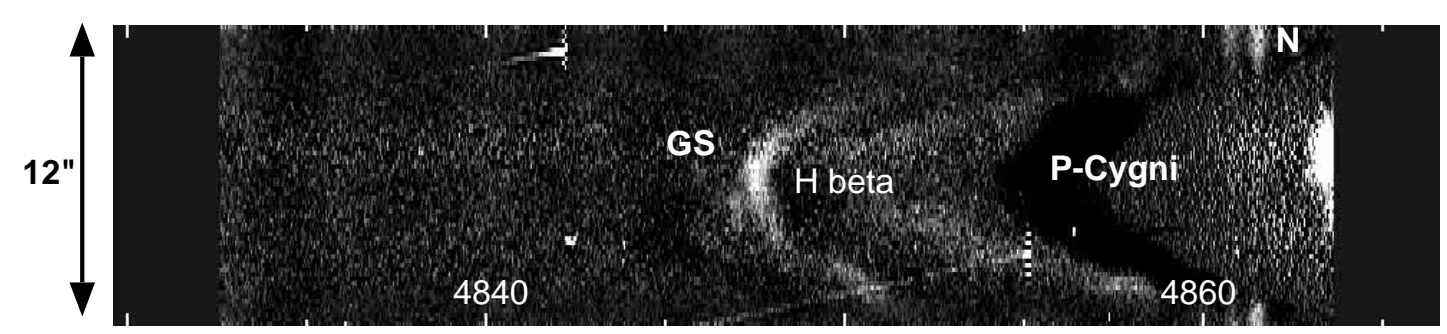

H beta

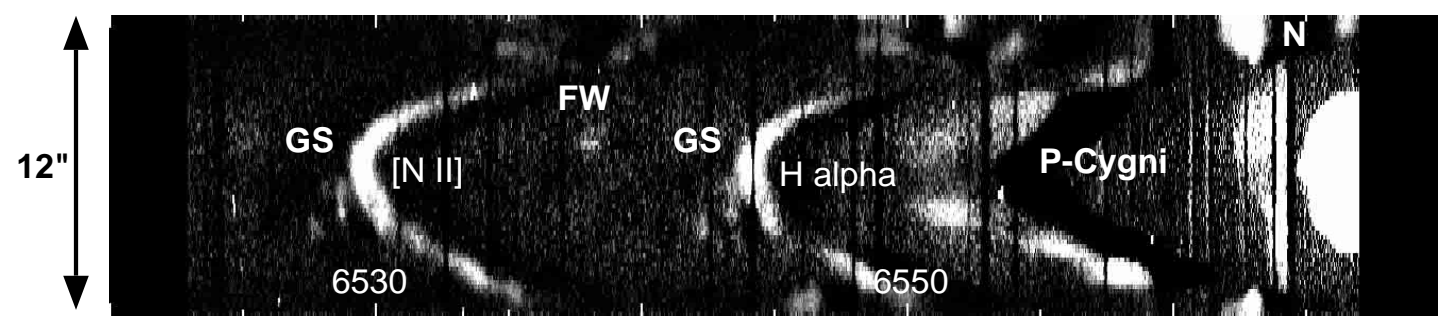

[N II] 6548

$\mathrm{H}$ alpha

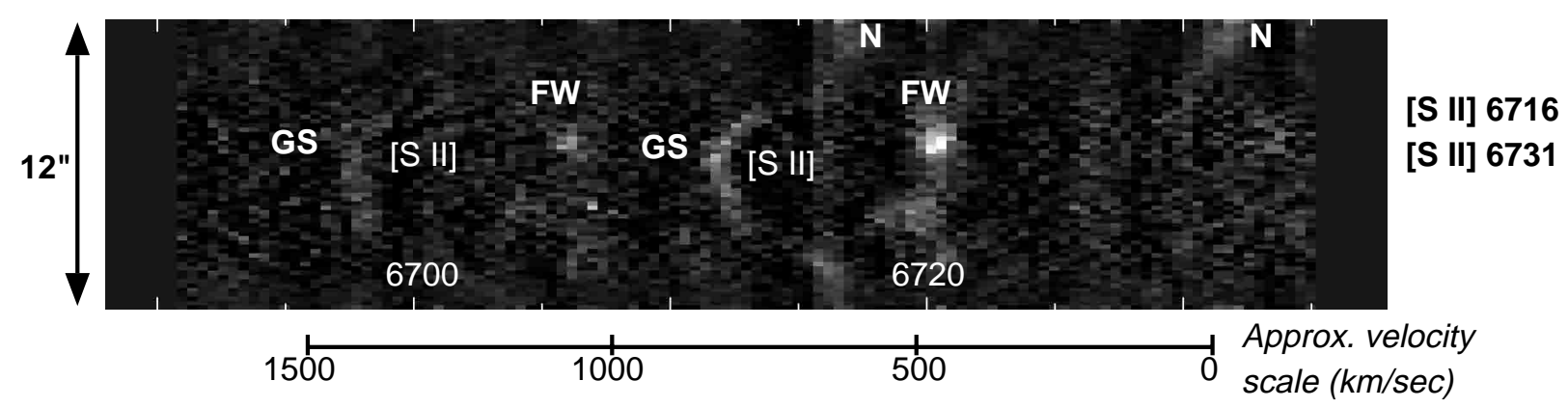

Fig. 2. Ghost Shell emission for five lines for slit -6. "GS" indicates Ghost Shell, "FW" Homunculus front wall, and "N" background nebular emission lines. $\mathrm{H} \alpha$ and $\mathrm{H} \beta$ P-Cygni features are noted. Images and velocity scale are aligned along zero-velocity reference wavelengths for $\mathrm{H} \beta, \mathrm{H} \alpha$, and [SII] 6730.8. Median subtraction has been applied to all images. The bottom image has been averaged over $0.4 \AA$ spectral bins to increase $S / N$.

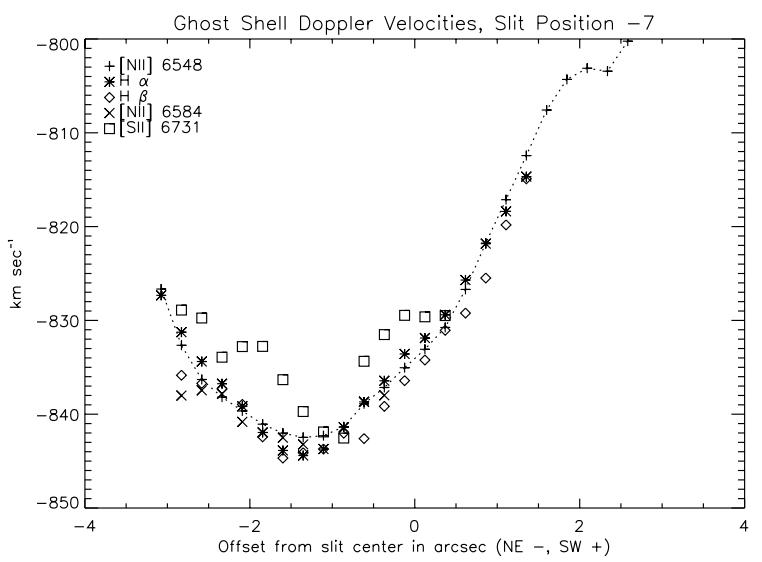

Fig. 3. Ghost Shell emission line Doppler velocities along slit -7. [NII] 6548 measurements are connected by dashed line.

a sphere of radius $\approx 11^{\prime \prime}$ that surrounds the Homunculus and possesses uniform radial velocity. We present three arguments based on the UVES observations to support this hypothesis: 1 ) observations in Slits $-6,-7$, and -8 , show that the GS emissions extend beyond the Homunculus spatial boundary, 2) analysis of the curvature of the GS lines in velocity space for individual slits yields an equivalent sphere of radius $\approx 11^{\prime \prime}$, and 3 ) assuming that the fast wind began during the Great Eruption epoch, and given the measured Doppler velocities for material behind the forward shock, we derive a radial velocity of $\approx 900 \mathrm{~km} \mathrm{~s}^{-1}$. Assuming that it is a heavy shock (see discussion below) we derive a velocity of the fast shock $\approx 1200 \mathrm{kms}^{-1}$. The product of this velocity with the elapsed time yields a current radius $\approx 12^{\prime \prime}$.

The first argument suggests that the GS lies outside of the Homunculus. The remaining arguments independently conclude that the GS has the form of a sphere of radius $11-12^{\prime \prime}$. Initial analysis of the data suggests that this sphere is centered along the AOS but somewhat (i.e., a few arcseconds) SE of the central star.

We emphasize that these results apply only to the $\mathrm{SE}$ lobe region beyond slit position -3 . The quasispherical approximation we propose is not valid near the central star and in the NW lobe region. In these other regions, the GS shell is distorted as it is penetrated by fast moving ejecta such as the equatorial disk and the north "jet" (vide Hester et al. 1991, for details of the debris field). We are continuing to analyze UVES data to determine the nature of the GS for these other regions. 
The details of the shape will be addressed in a publication in preparation.

\subsection{The Ghost Shell as the shock interface between fast and slow winds}

We propose that the Ghost Shell is the excited material associated with the forward shock created as the current fast wind propagates into the earlier slow massive wind. The rear shock has then swept up material to form the leading edge of the Homunculus. The wide separation between the GS and the forward edge of the Homunculus is due to the region being essentially adiabatic, a result of thermalizing a portion of the fast wind. However, the region emitting the Balmer, $[\mathrm{NII}]$, and $[\mathrm{SII}]$ lines is much cooler, as indicated by the line widths of these features and the $[\mathrm{SII}]$ temperature determination. This may be a result of cooling, or more likely, a mechanism similar to "Balmer shock", found in supernova remnants (Chevalier et al. 1980), although some puzzles remain when using this model. The approximately adiabatic conditions are confirmed by computations of the cooling times that are much longer than the dynamical times. The lack of agreement between the shape of the GS and the flask with the "dimple" of the Homunculus (Currie et al. 2000) may be due to the variation in density and/or other conditions of the slow wind as a function of latitude.

\subsection{Observation of Ghost Shell features in previous work}

Although the GS has never been identified as such, we believe that some features have been previously observed. Wallerstein et al. (2001) observed an unidentified emission line at $6530.8 \AA$, which coincides with the peak [NII] 6548 emission we observe when the UVES data are averaged over the SE lobe (i.e., slits -2 through -8). Gull \& Ishibashi (2001) reported $\mathrm{H} \alpha$ emission structures outside the Homunculus, some of which may correlate to the GS.

We have also detected a GS absorption complex in $\mathrm{CaH}$ and $\mathrm{K}$ bands for slit -3 in the 1999 data. This matches anomalous Ca absorption features reported by Davidson et al. (2001). The Doppler velocities Davidson et al. report for the $\mathrm{Ca}$ anomaly are generally consistent with the Doppler velocities we measure along the AOS for the GS emission lines.

\section{Conclusions and future work}

\subsection{Conclusions and implications}

We have discovered a unique new feature in the $\eta$ Carinae system, consisting of an emission nebula surrounding the SE lobe of the Homunculus. It generally has the appropriate emission lines for a fast shock (Chevalier et al. 1980) with the expected exception that we see no oxygen emission lines. We have also found a related set of absorption features in $\mathrm{Ca} \mathrm{K}$ and $\mathrm{H}$. The Doppler velocity, energy, and line width of the emission feature have been measured in three Balmer lines, two [NII], two [SII] lines, and [ArIII]. These measurements show great consistency (e.g., $\approx 1 \%$ for velocity measurements). We also argue that, over the SE lobe, the emission nebula roughly has the form of a spherical shell of radius $\approx 11^{\prime \prime}$. We conclude that the Ghost Shell represents the forward shock as the current fast wind, intially ejected during the epoch of the Great Eruption, propagates into the earlier slow massive wind.

\subsection{Future work}

Details of these arguments, including presentation of line strengths in absolute flux units, reddening corrections, discussion of the $S / N$ considerations, and extension of the analysis to the central star and NW lobe regions will be addressed in several papers now in preparation. Additional observations will be necessary in order to constrain the fully hydrodynamical modeling of the history. By combining observations with 3-D hydrodynamical modeling, we will explore new details of the Great Eruption and the evolution of both the forward shock (the GS) and the rear shock, that is, the Homunculus.

Acknowledgements. We wish to thank the European Southern Observatory for the opportunity to obtain these observations. Our results are possible due to the very high resolution and stability of UVES and the large collecting aperture of the VLT. The first author appreciates the support received from the ESO/Chile Visiting Scientist Program, and wishes to thank N. Langer, J. Stone, and F. Bacciotti for useful discussions. The second author wishes to thank A. Hajian for many helpful discussion and USNO for supporting the analysis and preparation activities associated with this paper.

\section{References}

Chevalier, R. A., Kirshner, R. P., \& Raymond, J. C. 1980, ApJ, $235,186 \mathrm{C}$

Currie, D. G., Dowling, D. M., Shaya, E. J., et al. 1996, AJ, 112,115

Currie, D. G., Le Mignant, D., Svensson, B., et al. 2000, ESO Messenger, 101, 24

Davidson, K., Smith, N., Gull, T. R., et al. 2001, ApJ, 121, 1569

Dowling, D. M. 1996, Ph.D. Thesis, Physics Dept., University of Maryland, College Park, MD, USA

Gull, T. R., \& Ishibashi, K. 2001, PASP, 242, 151

Hester, J. J., Light, R. M., Westphal, J. A., et al. 1991, AJ, $102,654 \mathrm{H}$

Humphreys, R. M., \& Davidson, K. 1994, PASP, 106, 1025

Wallerstein, G., Gilroy, K. K., Zethson, T., et al. 2001, PASP, 113,1210 